\title{
Development of a data analysis and performance evaluation system through the integration of Festo MPS stations
}

\author{
Eduardo Sallum, Student Member, IEE.
}

\begin{abstract}
Industria 4.0 is present in smart and digital manufacturing, making manufacturing companies improve productivity, reducing delivery time and related costs. The objective of this work is to demonstrate through three integrated MPS Festo stations (Distribution, Pick \& Place and Sorting), using the Internet of Things and Google Analytics technologies, the benefits in relation to remote performance monitoring. The intended objective is achieved through the implementation of the monitoring system at the three MPS Festo stations. The data obtained through the integration of the Festo stations and their respective sensors are processed and analyzed in a cloud infrastructure, so that the main metrics are visualized and transmitted on a panel. This monitoring system improves the perception of process performance, as the main performance metrics are displayed, such as productivity, cycle time and parts produced. The cloud infrastructure allows remote viewing and monitoring of the system.
\end{abstract}

Index Terms-MPS FESTO workstation, production management, cloud computing, process management, Android, iOS, RFID.

\section{INTRODUCTION}

$\mathbf{T}$ HE Internet of Things is the concept of everyday objects connecting to the Internet. Portable devices, vehicles, buildings, industrial machines and other items shipped with microelectronics, actuators and integrated sensors connected to the network allow, with the help of software, the exchange and collection of data forming an ecosystem [1].

The application of IoT to industry is known as Industrial Internet of Things (IIoT) or Industrial Internet. With smart objects connected, it allows more efficient access to the data collected from the manufacturing processes to the final product, generating unprecedented levels of efficiency, productivity and performance. Energy, oil, gas, health, food, automotive and aviation industries are some examples that are experiencing transforming operational and economic benefits. The Michelin Group, which in its industry uses sensors on the wheels of trucks to help drive drivers to save fuel, Taleris provides realtime data analysis to airlines to minimize disruptions caused by mechanical failures and delays and Tesla Motors, which in routine tests, found that the Model X had a defect in the front passenger seat that could cause airbag release anomalies and announced via email a recall for 4,000 vehicles to update the system software via over-the-air (OTA), without the need for the consumer to take the car to the workshop [2].

E. Sallum is with the Department of Informatics, Universidade Tecnológica Federal do Paraná, Ponta Grossa, PR. e-mail: esallum@gmail.com.
According to Ashton [3], the term Internet of Things (IoT) was first mentioned by him when he was part of MIT's Auto-ID Center in his work, "I made at Procter \& Gamble" in 1999. At that time, IoT was associated with the use of RFID (Radio-Frequency Identification) technology. According to Kelly (2013), the term Internet of Things became popular with the advance of Wireless Sensor Networks (RSSF) in industrial and residential automation in relation to energy use efficiency, latency, precision, fault tolerance and scalability. Things refers to a wide variety of connected objects, such as cardiac monitoring implants, automobiles with integrated sensors and DNA analysis devices for environmental monitoring.

The IoT allows objects that are uniquely identifiable with their embedded microelectronic system to be detected and controlled remotely through the network infrastructure, thus generating greater direct integration of the physical world with computerized systems and resulting in greater efficiency, accuracy and economic benefits [4].

The objectives of this work are:

(i) Emphasize general concepts of IIOT (Industrial Internet of Things), Big Data and AIDC technologies through Literature Review to demonstrate the importance of remote control and monitoring in an industrial environment;

(ii) Connect three MPS Festo teaching stations on a cloud monitoring platform (Google Cloud) by integrating Arduino with IOT devices (ESP8266 Wi-FI module);

(iii) Control MPS stations via RemoteXY mobile app (iOS and Android) via bluetooth connection (bluetooth module HC-05), showing real-time data on production time, quantity of parts produced, operating temperature and relative humidity.

(iv) Make the source code available to the community of all systems, such as the programming code in SCL language used on MPS Festo stations and the code in Arduino.

\section{RELATED WORK}

In this section we present related works that use the Festo MPS modular system as object of study.

The work of [5] presents the study of a pump control system in the compact Festo MPS workstation through the use of a fractional order controller, compatible with continuous (P, I, PI, PID) and batch controllers created in Simulink / Matlab. The results of the simulations show that the fractional order controller is related only to the phase margin, and not to the plant parameters. 
The work of [6] presents a remote performance monitoring system of the MPS Festo system through the integration of the Arduino platform, including temperature sensor and Wi-Fi module, with Google Analytics. Through this cloud monitoring, the user analyzes the system's performance through data collected such as productivity, cycle time and parts produced, and can intervene on the MPS Festo workstation in the event of an anomaly. Unlike the works presented, the proposed system allows the cloud monitoring of performance parameters such as productivity, time, temperature, humidity and number of parts and the control of MPS Festo workstations using iOS and Android mobile systems.

\section{LITERATURE REVIEW}

\section{A. Industrial Internet of Things (IIoT)}

IIoT is the interconnection of manufacturing-related processes using concepts applied to IoT. It is present in Industry 4.0 , which alludes to the application of Information Technology for industrial activities, incorporating machines, storage networks and manufacturing facilities in the form of physical cyber systems. This industrial revolution was preceded by three others: the first occurred with the introduction of the steam engine and the beginning of mechanical production; the second replaced steam with electricity, giving rise to the mass production assembly line with division of labor, and the third started the digital age, as it automated and robotized the production line through computerized systems and electronic circuits. Industry 4.0 comprises improvements obtained from the second and third industrial revolution [7].

IIoT objects present in manufacturing processes include intelligent industrial machines, automated storage systems and production facilities capable of exchanging data and making decisions independently. They improve the industrial processes involved in manufacturing, engineering, material use, supply chain and product life cycle management. With the new approach to production, smart objects are identifiable and localizable, in addition to having a data log with usage history and current status [8]. The essence of Industrial Internet of Things is composed of three elements:

(i) Connected industrial machines: uses IIoT concepts to connect sensors, actuators and embedded devices with microelectronics to industrial machines, integrating with PLCs and industrial process control applications. With this, data can be obtained, manipulated and transformed into information in real time;

(ii) Advanced analysis: optimizes the complexity of the algorithms to provide predictive analysis of control of production and manufacturing processes. With this analysis of physical phenomena of production processes, it is possible to understand and automate the operations of machines and industrial systems more efficiently. The benefits of this solution are favorable in several industrial processes, providing condition monitoring for the entire industrial process, preventive maintenance alerts and better energy management;

(iii) Connected people: provides with mobile devices greater access to information in real time, connectivity and intercommunication of people connected to the industry. Thus, it is permissible to optimize the development of industrial projects and operations, providing services of higher quality and safety [9].

IIoT provides greater financial additions for the industry, as it contributes to better flexibility and efficiency in the profitability of manufacturing processes. New technologies embedded in digital sensors allow wireless communication with protocols and the use of batteries as a power source, increasing the number of industrial projects and equipment that can be converted to IIoT. Connecting devices, facilities, fleets and smart grids to industry individuals provides process optimization solutions and unlocks the potential for productivity development [10].

\section{B. Big Data}

Big Data allows you to take advantage in a highly competitive industrial environment. The challenge of Big Data is to find the necessary set of skills, tools, techniques and resources required to deal with the complexity arising from the huge amount of data generated by Industry 4.0. In the literature, Big Data is represented through 4 concepts:

(i) Volume: Consists of the amount of data generated and stored. The size of the data determines value and the potential to transform it into information. In addition, the volume of data decides whether the Big Data concept can be applied to a given data set or not;

(ii) Variety: The type and nature of data are varied, such as: images, texts, videos, audios, etc. Consequently, the complexity of analyzes to generate increases. The advantages of having a wide variety of data obtained from the same process are applying data fusion techniques to fill 'missing pieces' between one analysis and another;

(iii) Speed: The data entered in the context of Big Datas are produced more continuously in relation to the small data set. Due to the large volume and variety of data, the processing for data to be generated and processed to meet the demands of an industry must be fast;

(iv) Veracity: It is the extended definition for Big Data, which refers to the quality, value and usefulness of the data obtained [11].

The data collected and analyzed with mining techniques allow routine activities to be carried out with the aim of inspecting and testing the presence of warning conditions that indicate that a component is about to fail. With this, it is possible to schedule a correlative maintenance to replace, repair or overhaul the component before the predicted failure, and in some cases eminent, minimizing risks of production delay [12].

\section{AIDC technologies}

AIDC (Automatic Identification and Data Capture) technologies, also known as Auto-ID, refer to the methods of automatic identification of objects present in the IoT, collecting data and inserting them directly into a database. Technologies considered as part of it include barcodes, QR-Code (QuickResponse Code), NFC (Near Field Communication) and RFID, 
acting as the basis for automated data collection, identification and analysis systems worldwide. They found their importance in several industrial processes, including semiconductor manufacturers such as Motorola, which started using RFID in aseptic rooms in order to increase quality control and efficiency in the use of equipment. It is also used in industrial vehicle automation systems for the ability to track moving objects [13]. The tags of a Passive RFID operating in UHF (Ultra High Frequency) in ideal conditions can reach more than 30 meters of reading distance. Active RFID ranges have a range of hundreds of meters of reading distance, but to conserve battery life they can be configured for a lower transmission power, reaching about 100 meters [14].

\section{Methodology}

\section{A. MPS Festo workstations}

For this work, we used three didactic workstations MPS Festo: MPS Distribution station, Pick \& Place Station MPS, and MPS Sorting Station.

1) MPS Distribution station: The MPS Distribution station is responsible for pushing the parts that are stored in the stacking magazine tube on the production line. The double-acting cylinder is activated to place the pieces, one at a time, on the conveyor. In this system, the Conveyor module transports the workpiece to the right [15]. Figure 1 shows the MPS Distribution workstation:

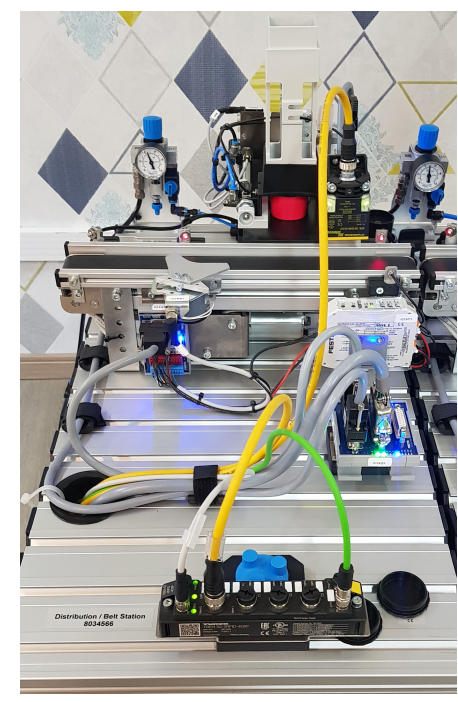

Fig. 1. MPS Distribution.

2) Pick \& Place MPS Station: The MPS Pick \& Place station is responsible for inserting the cover on the workpiece, through a module called Pick \& Place with two axes. Diffuse optical sensors detect the arrival of the part, coming through the distribution station, and initiate the conveyor module for the movement of the part so that it is covered. The Pick \& Place module takes a workpiece insert from the ramp and places it in the workpiece compartment. The complete workpiece (housing and insert) is released by the separator and transported to the end of the conveyor [16]. Figure 2 presents the MPS Pick \& Place workstation:

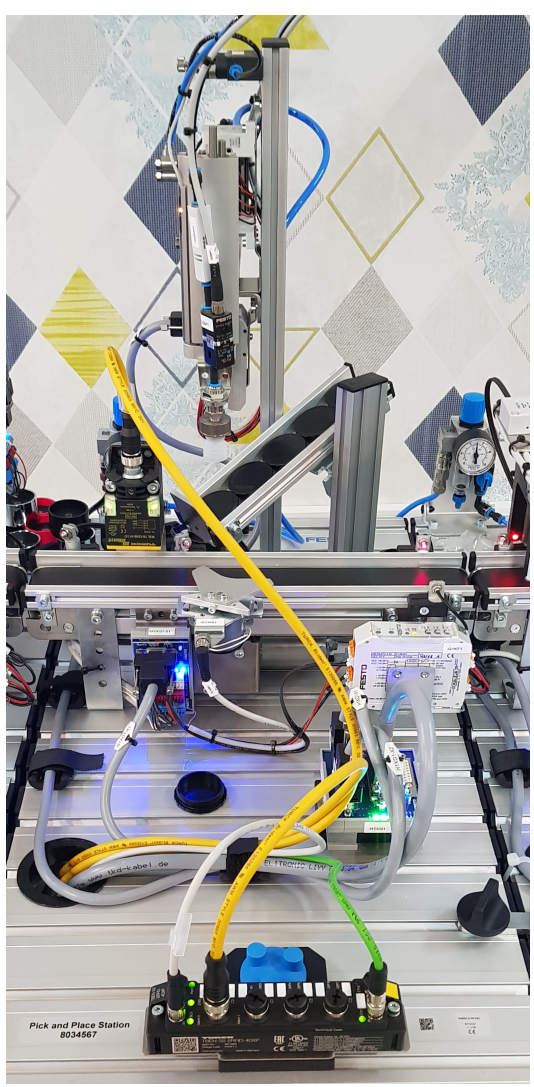

Fig. 2. MPS Pick \& Place MPS.

3) Sorting MPS Station: This station classifies the pieces on three slides. The parts placed at the beginning of the conveyor are detected by a diffuse sensor. A pneumatic stopper with an integrated valve interrupts the part before the sorting process. The sensors upstream of the stopper detect the features of the part (black, red, metal). Cylindrical parts are classified on the appropriate slides using electrical deflectors. A retro-reflective sensor monitors the slide fill levels. Inductive and optoelectric sensors detect the color and material of the parts. A short stroke cylinder for parts on the moving conveyor and passes them through for classification on one of the three slides [17]. Figure 3 shows the MPS Sorting workstation: 


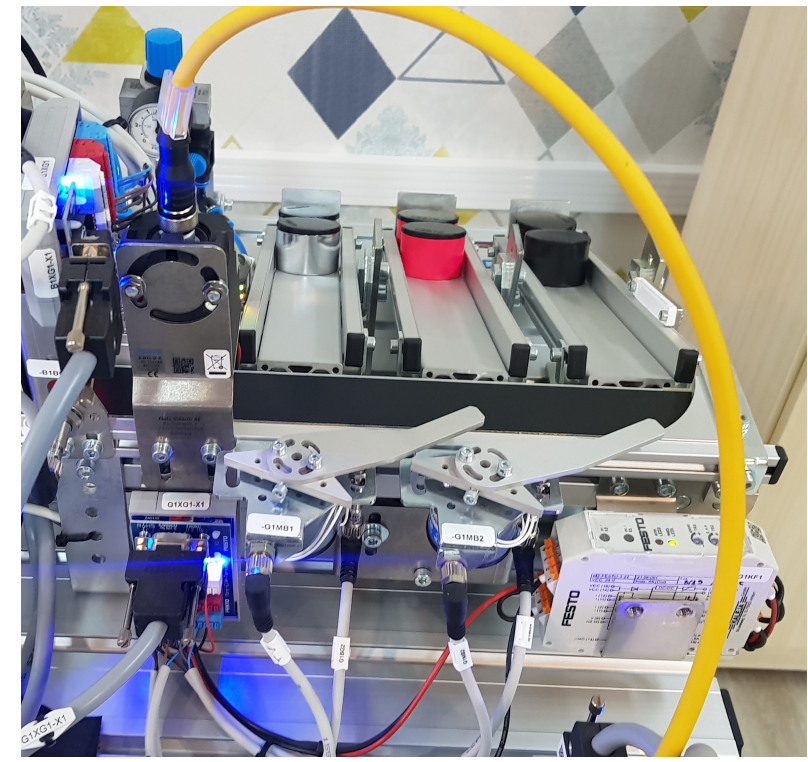

Fig. 3. Sorting MPS

Figure 4 shows the three integrated MPS Festo workstations

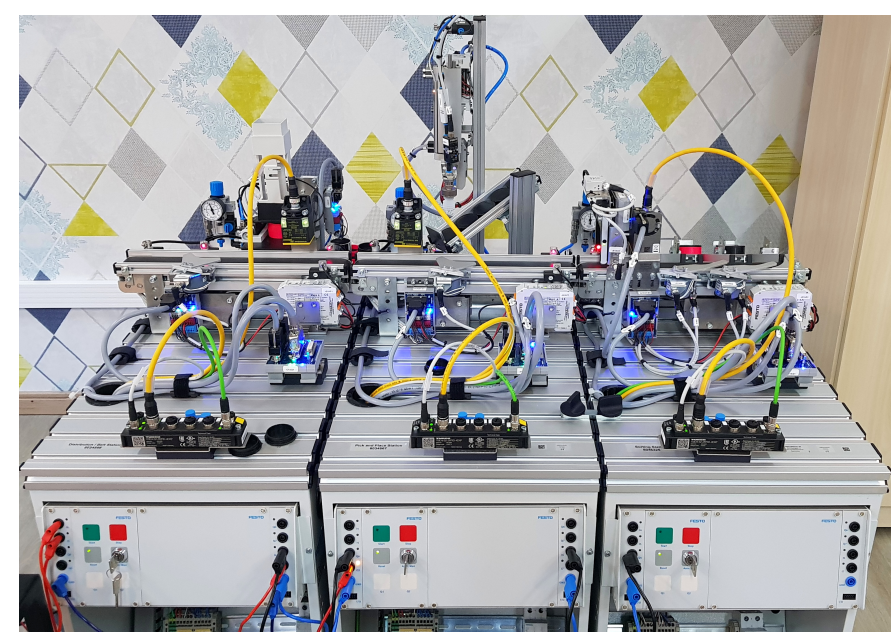

Fig. 4. Three integrated workstations: MPS Distribution (left), Pick \& Place MPS (center), and Sorting MPS (right).

\section{B. Mobile control system}

Figure 5 illustrates the scheme used in this work:

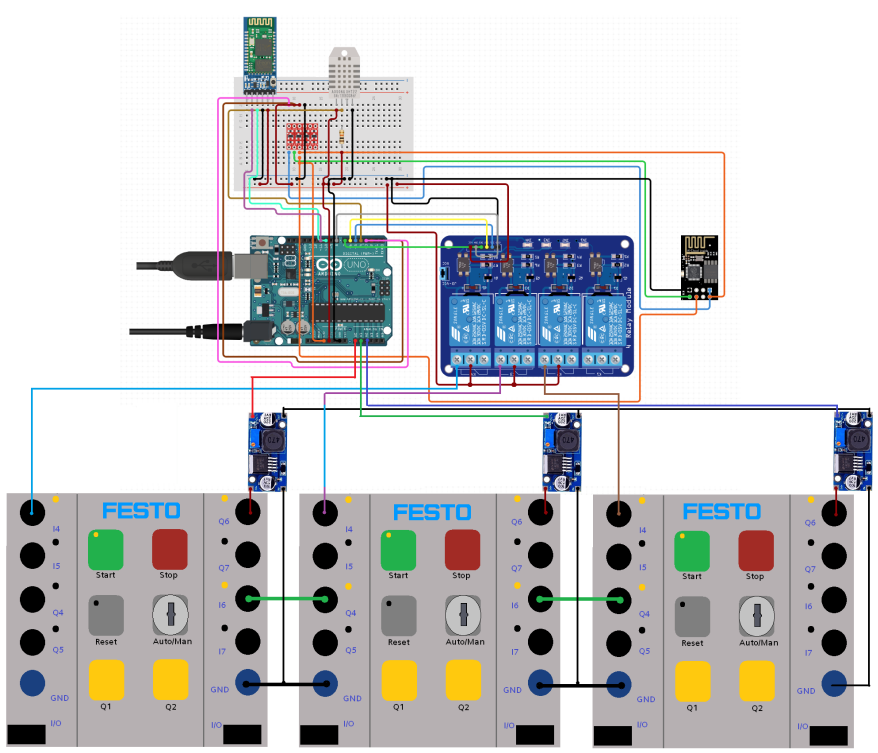

Fig. 5. Electronic scheme.

According to Figure 5, the electronic scheme consists of:

1) Arduino Uno: Is an open-source project based on the ATmega328P microcontroller. The Arduino UNO board has 14 pins that can be used as digital input or output. These pins operate at $5 \mathrm{~V}$, where each pin can supply or receive a maximum current of $40 \mathrm{~mA}$. Each pin has an internal pull-up resistor that can be enabled by software. Figure 6 shows the Arduino Uno:

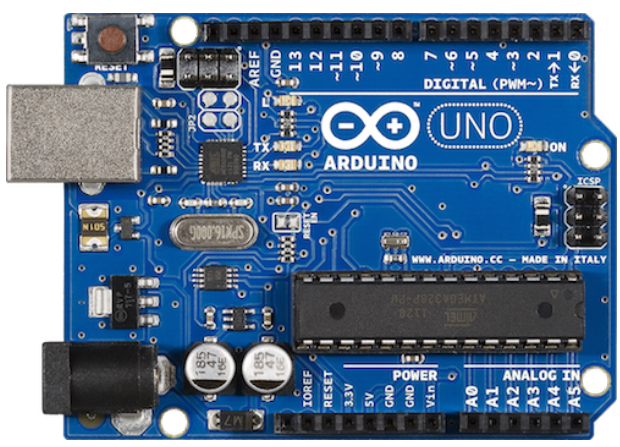

Fig. 6. Arduino Uno [18].

2) Festo MPS control panel: The control panel is connected to the controller via SysLink and allows up to 16 inputs and 16 operational outputs to be connected. In this project, the Start button starts the operation of the respective workstation. The Stop button for operating all moving parts of the workstation and the Reset button returns the workstation to its initial condition. Figure 7 shows the control panel design of the Festo stations: 


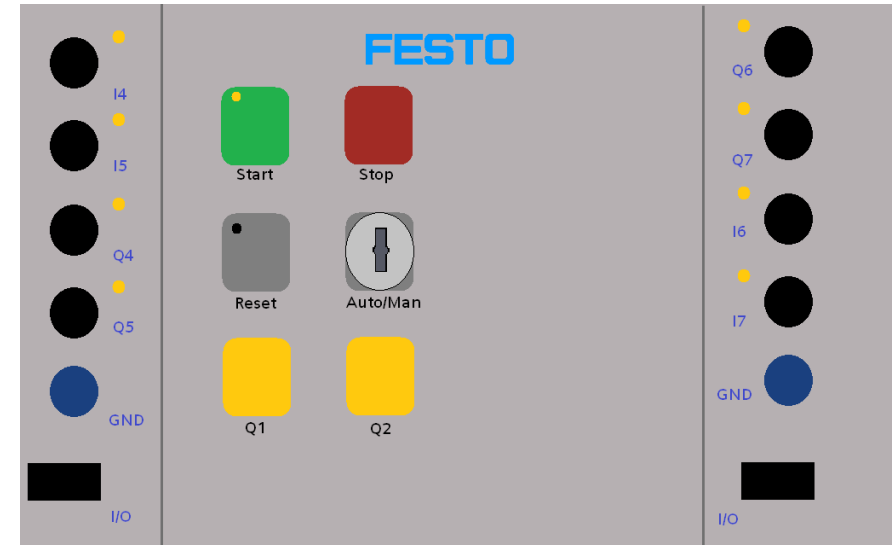

Fig. 7. Festo MPS control panel.

3) Wi-Fi and Bluetooth module: The Wi-Fi module used is ESP8266, which allows the connection of Festo workstations with the remote cloud monitoring platform. The HC-05 bluetooth module connects workstations with iOS and Android mobile devices, allowing the user to control the panel of the 3 workstations remotely. Figure 8 presents the ESP8266 Wi-Fi module and the HC-5 bluetooth module, respectively.

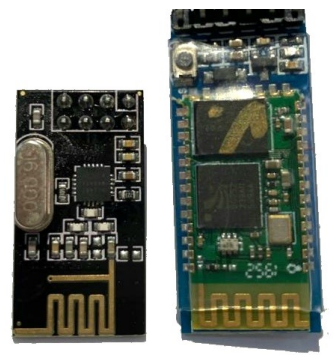

Fig. 8. ESP8266 Wi-fi module (on the left) and the HC-5 bluetooth module (on the right).

4) Voltage regulator module LM2596: The LM2596 voltage regulator works as a DC/DC converter in Step Down mode, being able to reduce a load of up to $3 \mathrm{~A}$ with optimum efficiency. The output voltage can be adjusted between 1.5 to $35 \mathrm{v}$, with input 3.2 to $40 \mathrm{v}$. It is connected to the Q6 outputs of the MPS Festo control panels of the three Festo MPS. Figure 9 presents the the LM2596 voltage regulator:

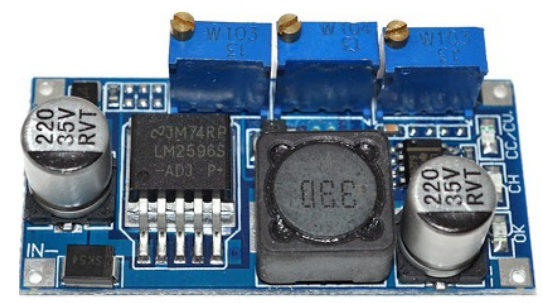

Fig. 9. LM2596 voltage regulator [19].

5) RemoteXY application: The RemoteXY application allows you to create and use an interface for the control of controller cards via smartphone or tablet through a graphical interface editor. The interface structure of the RemoteXY mobile application is stored on the Arduino. The main advantage of RemoteXY is that the control interface structure is sent to the mobile device via Arduino via a bluetooth connection, without the need to connect to external servers to download the control interface.

\section{Cloud monitoring system}

The cloud monitoring system monitors the following variables:

\section{- Productivity:}

- Production time of piece by color ;

- Quantity of part production per color ;

- Average part productivity per color (parts per minute);

- Quality control:

- Real-time ambient temperature and relative humidity.

- Maintenance of stations:

- Running time of the belts of each station;

- Time of operation of the distribution magazine (doubleacting cylinder);

- Operating time of the Pick \& Place module.

\section{- Other data:}

- Full slide (Sorting);

- Number of "full slide" events;

- Slide time full (metric to see the time the production was stopped);

- Number of pieces lost (Number of pieces distributed by the Distribution stations minus the number of pieces classified by the Sorting station).

The Average Production, represented in number of parts per minute, is calculated using Equation 1:

$$
\text { Average Productivity }=\frac{\text { total number of processed parts }}{\text { total working time }}(1)
$$

\section{Cloud monitoring platform}

The monitoring platform used is AskSensors ${ }^{1}$, which allows you to remotely monitor sensors and control actuators, manage connected devices in real time and analyze data acquired in the cloud.

\section{RESULts}

Through the RemoteXY application and the Asksensors platform, it was possible to control and monitor the 3 MPS Festo workstations. Figures 10 and 11 show the dashboar for monitoring air temperature and relative humidity, respectively.

\footnotetext{
${ }^{1}$ https://asksensors.com/
} 


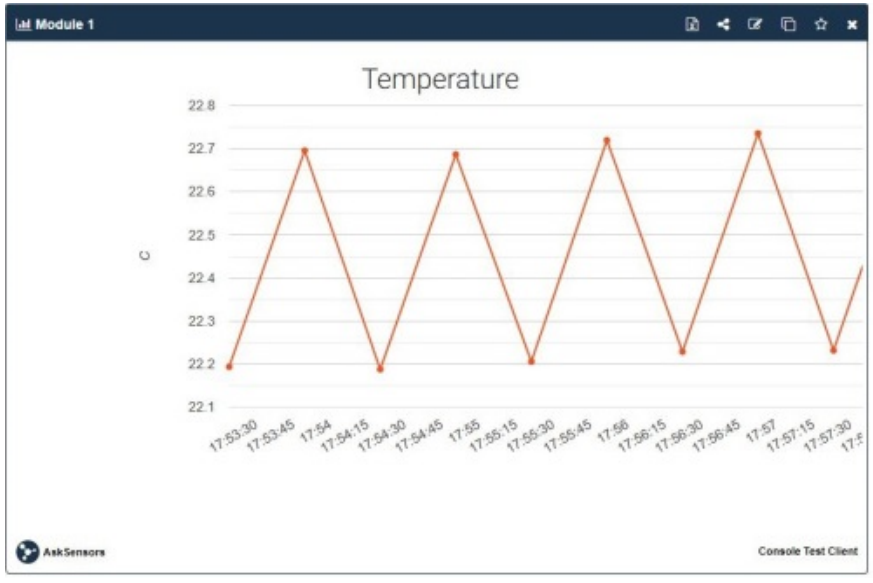

Fig. 10. Ambient Temperature Monitoring

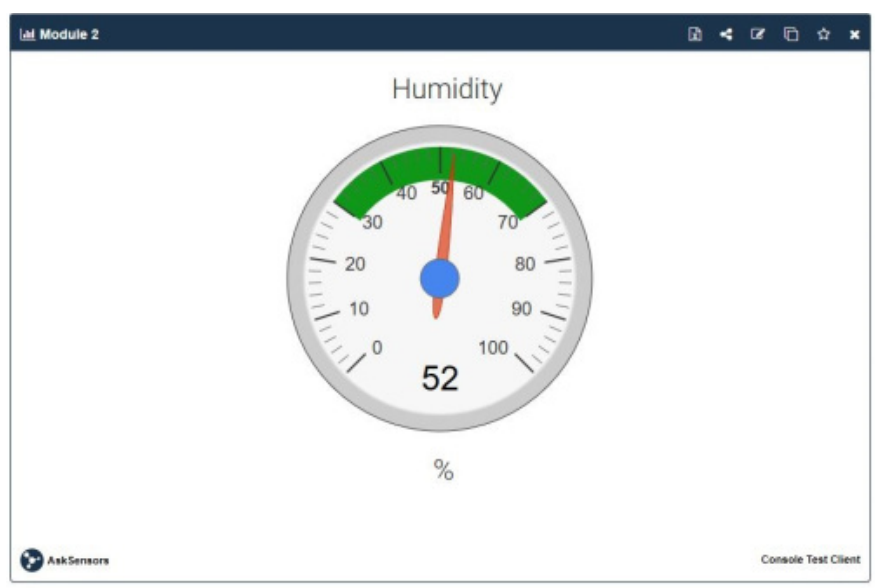

Fig. 11. Ambient Humidity Monitoring

Figure 12 shows the RemoteXY application interface on a mobile device:

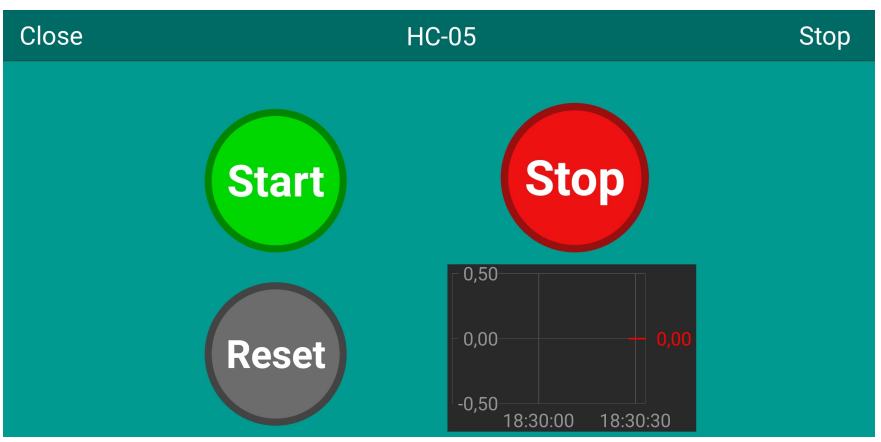

Fig. 12. RemoteXY application

As shown in Figure 12, it is possible to control the panel of the three MPS Festo stations through the mps festo mobile app. The choice to control the three integrated stations is to provide a simpler and more practical user interface. Additionally, the user visualizes the graph of parts produced by time.

\section{CONCLUSIONS AND FUTURE WORK}

IIoT integrates devices equipped with detection, identification, processing, communication and network resources. Sen- sors and actuators are getting smaller and smaller, with greater processing power, coupled with the growing demand for RFID devices, making it easier to monitor and control industrial machines. IIoT's object connection optimizes processes and increases industry productivity, providing a promising opportunity for building powerful industrial systems and applications. IIoT benefits the entire industrial chain, from manufacturing to the final consumer, providing better production planning, control and tracking, reducing costs and increasing decisionmaking capacity.

For future work, we will add more sensors to our MPS Festo workstation control and monitoring system in order to control the operating temperature of the mobile parts of the workstations and the production environment of the parts.

\section{REFERENCES}

[1] J. Gubbi, R. Buyya, S. Marusic, and M. Palaniswami, "Internet of things (iot): A vision, architectural elements, and future directions," Future generation computer systems, vol. 29, no. 7, pp. 1645-1660, 2013.

[2] P. Daugherty, P. Banerjee, W. Negm, and A. E. Alter, "Driving unconventional growth through the industrial internet of things," accenture technology, 2015.

[3] K. Ashton et al., "That 'internet of things' thing," RFID journal, vol. 22, no. 7, pp. 97-114, 2009.

[4] F. Mattern and C. Floerkemeier, "From the internet of computers to the internet of things," in From active data management to event-based systems and more. Springer, 2010, pp. 242-259.

[5] A. Koszewnik, T. Nartowicz, and E. Pawłuszewicz, "Fractional order controller to control pump in festo mps ${ }^{\circledR}$ pa compact workstation," in 2016 17th International Carpathian Control Conference (ICCC), 2016, pp. 364-367.

[6] F. Giusti, M. Bevilacqua, S. Tedeschi, and C. Emmanouilidis, "Data analytics and production efficiency evaluation on a flexible manufacturing cell," in 2018 IEEE International Instrumentation and Measurement Technology Conference (I2MTC), 2018, pp. 1-6.

[7] K. Bloede, G. Mischou, A. Senan, and A. Tilow, "The industrial internet of things: making factories "smart" for the next industrial revolution," in Woodside Capital Partners, 2017.

[8] H. Kagermann, J. Helbig, A. Hellinger, and W. Wahlster, Recommendations for implementing the strategic initiative INDUSTRIE 4.0: Securing the future of German manufacturing industry; final report of the Industrie 4.0 Working Group. Forschungsunion, 2013.

[9] P. C. Evans and M. Annunziata, "Industrial internet: Pushing the boundaries," General Electric Reports, pp. 488-508, 2012.

[10] H. Yang, S. Kumara, S. T. Bukkapatnam, and F. Tsung, "The internet of things for smart manufacturing: A review," IISE Transactions, vol. 51, no. 11, pp. 1190-1216, 2019.

[11] M. Hilbert, "Big data for development: A review of promises and challenges," Development Policy Review, vol. 34, no. 1, pp. 135-174, 2016.

[12] J. Lee, H.-A. Kao, S. Yang et al., "Service innovation and smart analytics for industry 4.0 and big data environment," Procedia Cirp, vol. 16, no. 1, pp. 3-8, 2014.

[13] L. S. Smith, "Rfid and other embedded technologies who owns the data," Santa Clara Computer \& High Tech. LJ, vol. 22, p. 695, 2005.

[14] Y.-L. Chen, D. Liu, S. Wang, Y.-F. Li, and X.-S. Zhang, "Self-powered smart active rfid tag integrated with wearable hybrid nanogenerator," Nano Energy, vol. 64, p. 103911, 2019.

[15] "Mps distributing station - basic principles of mechatronic systems," https://www.festo-didactic.com/br$\mathrm{pt} / \mathrm{mps}$-sistema-modular-de-producao/estacoes/mpsdistributing-station-basic-principles-of-mechatronicsystems.htm?fbid=YnIucHQuNTM3LjIzLjE4LjYwNi44MTQw, Festo, Jul 2020.

[16] "Pickplace station - small is beautiful," https://www.festo-didactic.com/br-pt/mps-sistema-modular-deproducao/estacoes/8149.htm?fbid=YnIucHQuNTM3LjIzLjE4LjYwNi44MTQ5, Festo, Jul 2020 
[17] "Mps sorting station d - combining opto and inductive sensors," https://www.festo-didactic.com/br-pt/mps-sistema-modular-de-

producao/estacoes/mps-sorting-station-d-combining-opto-and-inductivesensors.htm?fbid=YnIucHQuNTM3LjIzLjE4LjYwNi44Mjk1, Festo, Jul 2020.

[18] "Arduino uno rev3," https://store.arduino.cc/usa/arduino-uno-rev3, Arduino, Sep 2020.

[19] "Lm2596 module sensors for arduino," http://www.arduinostarterskit.com/sale-10208670-dc-dc-step-downpower-module-lm2596-module-sensors-for-arduino-7v-35v-to-1-25v30v-3a.html, OKY NEWSTAR TECHNOLOGY CO, Sep 2020. 